\title{
COLPOCLEISIS DE LE FORT
}

REVISION DE 10 AÑOS EN EL SERVICIO DE GINECOLOGIA Y OBSTETRICIA DEL HOSPITAL SAN JUAN DE DIOS DE BOGOTA

\section{Laureano Marin Ardila*}

Teniendo en cuenta que muchas de las pacientes de edad avanzada, asocian en su senectud, el prolapso genital a condiciones múltiples de patología médica, he querido hacer un análisis de los resultados obtenidos con la práctica de la Colpocleisis de Le Fort, en 53 casos que corresponden a la revisión de los últimos 10 años en el servicio de Ginecología y Obstetricia del Hospital San Juan de Dios de Bogotá, donde mi participación fué activa en aproximadamente la tercera parte.

Como todos sabemos muchas pacientes en estas condiciones, son o fueron tratadas paliativamente con pesarios, pero como éstos deben ser removidos frecuentemente, para asearlos o por intolerancia, irritación de la mucosa vaginal o cervical, falta de acomodación, relajación del piso pélvico, uso prolongado, factor psicológico, etc., es por lo que se hace necesaria una intervención, que a la vez que corrija el prolapso, sea lo más inocua posible, no solo desde el punto de vista quirúrgico sino también anestésico.

Ya que mi pensamiento concuerda en que la Colpocleisis de Le Fort es

* Depto, de Ginecología y Obstetricia - Facultad de Medicina, U. Nal. Colombia. la cirugía más llamada para estos casos y en los últimos años poco se ha escrito sobre ella, voy a traer a cuento una pequeña reseña histórica.

Fue en 1823 cuando Jerardín insinuó que el prolapso genital en estas pacientes seniles podía corregirse recortando y suturando la mucosa vaginal. En 1867 Neugebauer siguiendo las insinuaciones del anterior sí practicó la intervención, siendo León Le Fort quien en 1876 la describió con mínimas modificaciones, llamándola Colpocleisis o Colporrafia Media. Este autor discutiendo el mecanismo del prolapso decía que antes que el útero, desciende la mucosa vaginal, de lo cual dedujo que el prolapso se reduce guardando las paredes vaginales anterior y posterior en aposición. También pensó que un periné relajado no podía favorecer un resultado satisfactorio, por lo que su primera operación la realizó en dos tiempos, practicando a los ocho días la perineorrafia.

En principio la técnica no fue muy aceptada, pero luego se reintrodujo en EE. UU. por Berlín en 1880, quien reportó tres casos, fallando uno, al cual no le practicó perineorrafia.

Desde esa época el procedimiento ha sufrido pequeñas modificaciones, 
principalmente en relación con la dimensión del rectángulo a resecar en la mucosa vaginal.

En 1889 Taft, reportó un caso y su comentario fue de que la técnica no parecía muy fácil. En 1912 Wyatt, señaló ocho intervenciones realizadas por el Dr. Tate, quien usó catgut cromado en la sutura. Gotte, en 1922 reporta 12 operaciones de Le Fort que realizó con anestesia raquídea, obteniendo excelentes resultados, haciendo énfasis en que la colpectomía parcial era mucho menos grave que la total.

Brock y Mora, en 1825, reportaron 39 casos, sin serias complicaciones. En 1934, Bellas también habló de la colpocleisis, pero no mencionó el número de casos por él practicados. Phaneuf, en 1935, señaló 20 casos con dos recidivas. Adair y $\mathrm{Da}$ Sef, ci$\tan 38$ casos con una falla. Collins y Lok, en 1941, anotan 31 casos con resultados satisfactorios en 29 de ellos. Mazer e Israel, en 1948, hicieron un análisis de 33 casos, presentando 12 de ellos cervicitis, por lo que primero practicaron amputación circular de cuello y después la colpocleisis.

Wolf, presentó 14 casos en 1952, con buenos resultados sugiriendo que esta intervención debería practicarse con más frecuencia.

Goodall y Power imprimieron una modificación a Le Fort la cual permitía efectuar el acto sexual. Estos autores practicaron la denudación de la mucosa vaginal anterior $y$ posterior en forma triangular, creando una doble vagina en el tercio superior y permaneciendo simple en los dos tercios inferiores del canal vaginal (8).

\section{MATERIAL Y METODO}

Fueron revisadas 53 historias de pacientes a las que se les practicó col- pocleisis de Le Fort y que corresponden a los 10 últimos años de labor, (1956 a 1965) en el departamento de Ginecología y Obtetricia del Hospital San Juan de Dios de Bogotá. Estas intervenciones fueron realizadas por el personal docente y el adiestramiento de esta institución. El método seguido es el estadístico.

\section{RESULTADOS Y COMENTARIOS}

Fueron seleccionadas las pacientes de más avanzada edad con prolapso genital grado III, de cuello restante, de cúpula vaginal, y una con Elongatio Coli agregado, todas sin vida sexual activa y post-menopáusicas.

Fueron síntomas constantes, la sensación de cuerpo extraño a través de vagina, la frecuencia urinaria, disuria e incontinencia urinaria de esfuerzo.

Edad. Esta osciló entre 55 y 88 años, siendo el mayor número de pacientes afectadas, las comprendidas entre los 70 y los 75 años, como lo indica la gráfica adjunta. El promedio de edad en las 53 pacientes fue de 72.83 .

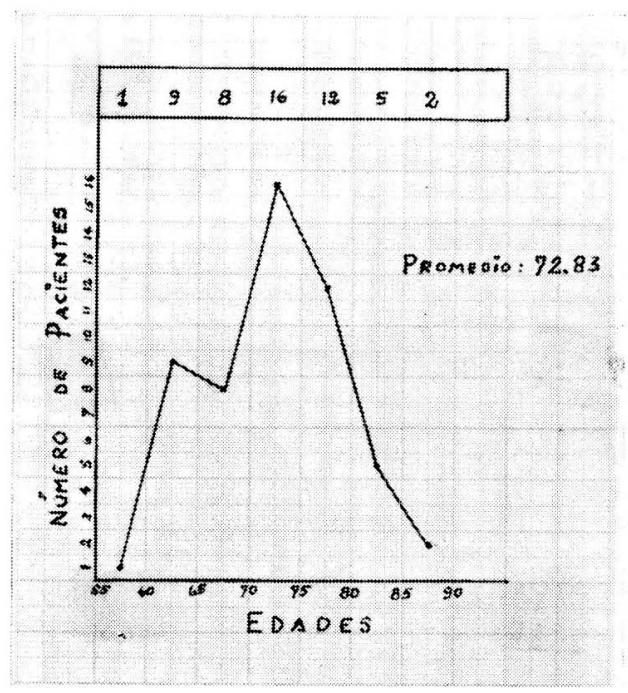


La paciente de 55 años, que en realidad es un caso excepcional por la colad para este tipo de cirugía antifisiológica, presentó un riesgo quirúrgico aumentado a causa de su problema cardiovascular y pulmonar, que hicieron imperativo buscar una técnica anestésica y quirúrgica lo menos traumática posible y tan buena como la que más, a fin de asegurar una supervivencia post-operatoria.

Paridad. Se hallaron pacientes desde nuligestantes hasta grandes multíparas, comprendiendo la gran mayoría multíparas entre 4 y 7 embarazos.

Como dato curioso he querido hacer notar la relación de aumento entre el número de gestaciones con el número de abortos. La curva de gestaciones creció notablemente entre 4 y 7 embarazos, correspondiendo exactamente al mismo aumento en frecuencia de abortos.

El cuadro representado en la gráfica muestra la paridad repartida en grupos de tres, para mejor relación con el número de casos.

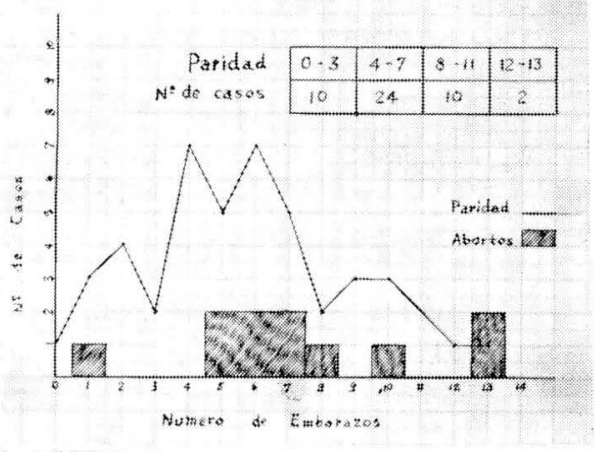

Diagnóstico ginecológico. Todas presentaron prolapso genital grado ill, uno de cuello restante, dos de cúpula vaginal y una con Elongatio
Coli sobreagregado. El dato de desgarro perineal y enterocele no son constantes. La deficiencia en la confección de algunas historias no permite una mejor recolección de datos.

\section{TIEMPO DE EVOLUCION}

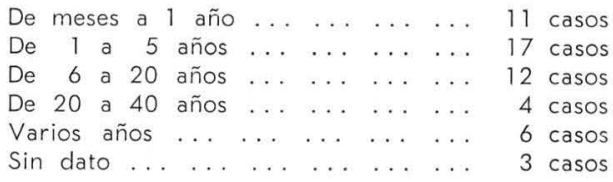

Este cuadro es muy demostrativo del abandono que de su salud hacen nuestras gentes de clientela hospitalaria, especialmente en lo relacionado al aparato genital femenino. Aparece de bulto la relación del tiempo que estas mujeres habían soportado las molestias inherentes a su lesión genital, pues no es de pasar por alto anotar que la inmensa mayoría llevaron consigo la enfermedad por varios años y 4 de ellas por espacio de 20 a 40 años, tiempo increiblemente largo para soportar tal incomodidad. En realidad un pequeño número, 11 de todos los casos que corresponde a un $20.7 \%$ consultaron en el primer año de la enfermedad.

Diagnósticos asociados al prolapso genital. Para un mejor orden en el estudio, me he permitido organizarlos como lo demuestran los cuadros que a continuación expongo.

\section{CARDIOVASCULAR}

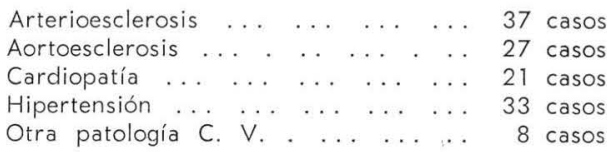

PULMONAR $Y$ VIAS AEREAS ALTAS

Enfisema pulmonar ... ......... 34 casos Bronquitis ... ... . . . . . . . . 7 casos Esclerosis pulmonar ... ... .... . . 2 casos Sinusitis ... . . . . . . . . . . . . 3 casos

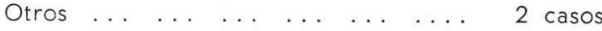

Es sin lugar a dudas la patología cardiovascular y pulmonar de curso 
severo, la que ha justificado más veces este tipo de cirugía. Gracias a los adelantos conseguidos en la anestesia, en el manejo de los electrolitos, a la destreza del cirujano, etc., es lo que ha permitido favorecer a estas pacientes de edad avanzada con esta cirugía cuya sencilla y fácil ejecución no ofrece riesgo operatorio muy crecido.

\section{PATOLOGIA GINECOLOGICA ASOCIADA}

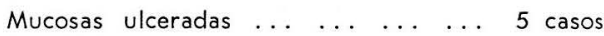

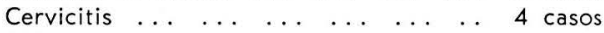

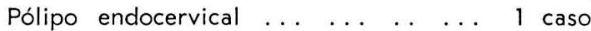

Esta patología ginecológica asociada no interrumpe para nada la práctica de la intervención, pero sí obliga en una forma más decidida a buscar con estudios minuciosos correspondientes, la presencia de un posible proceso maligno que no sería perdonable pasar desapercibido. Sobra decir que la preparación de la mucosa debe lograrse con el tratamiento médico correspondiente para un mejor éxito de la operación, si los resultados de los análisis la autorizan.

\section{OTRA PATOLOGIA}

Bocio multinodular ... ... ...

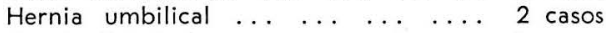

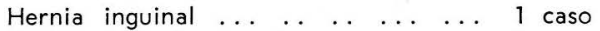
Eventración abdominal ... $\ldots . . . . \quad 1$ caso Procidencia de recto grado IV ... .. 2 casos Desnutrición ... ... . . . . . . . . . 1 caso

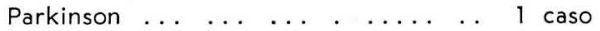

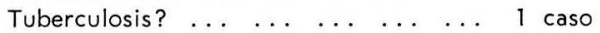

En los casos de procidencia de recto grado IV se practicó la intervención conjunta con el Proctólogo, siendo estas dos pacientes las que presentaron un post-operatorio más largo. De todos modos esta otra patología mencionada en el cuadro, fue de menos valor en el riesgo de la intervención.

Concepto cardiovascular. Lo he clasificado de acuerdo con el riesgo quirúrgico que ofrece, en la siguiente forma:

Riesgo normal . . 20 casos para un $43.4 \%$ Riesgo moderado . . 11 casos para un $23.9 \%$ Riesgo aumentado . . 14 casos para un $30.4 \%$ Sin riesgo ... . . 1 caso para un $2.1 \%$

Estos datos se obtuvieron sobre 46 historias que contenían el concepto cardiovascular y que corresponden al $86.8 \%$. Sin dato 7 historias para un $13.2 \%$. Como puede observarse todas las pacientes menos una, prescntaron algún riesgo quirúrgico, lo que demuestra el interés de este tipo de cirugía para pacientes ancianas.

Citología. Solo se obtuvo en 19 historias, siendo en todas negativa para carcinoma. (Aclaro que las citologías solo se vienen practicando de rutina en este servicio desde 1963). Es una lástima que estos exámenes no se hayan practicado en todas las pacientes, pues los considero imperativos como medio de colaboración en el diagnóstico de carcinoma.

Laboratorio. Este no demostró alteraciones dignas de mención antes ni después de la intervención.

Anatomía patológica. Estos datos solamente se encontraron en 36 historias, algunas con dato pre o postoperatorio y otras con ambos así:

Con dato: 36 casos para un $67.9 \%$ Sin dato : 17 casos para un $32.1 \%$

\section{RESULTADOS PRE-OPERATORIOS}

Cervicitis ... ... . . . . . . ... . . 7 casos

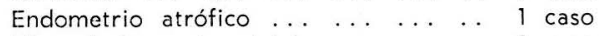

Hiperplasia endometrial ... ... . . 1 caso

\section{RESULTADOS POST-OPERATORIOS}

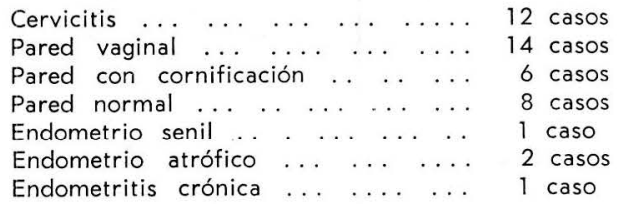

Preparación pre-operatoria. Es norma en el servicio, hospitalizar la pa- 
ciente quirúrgica libre de cualquier proceso asociado agudo que dificulte su pronta intervención, excepto casos especiales. Aquellas que requerían tratamiento previo y hospitalario fueron tratadas así:

Antibióticos, expectorantes,

broncodilatadores ... ... . ..... 15 casos

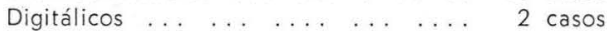

Mecha ictiolada ... ... ... .... 2 casos

Estrógenos ... . ... ... . .. 4 casos

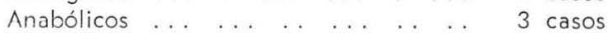

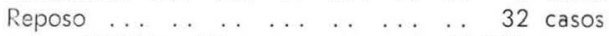
TOTAL: 32 casos para un $60.3 \%$

Anestesia utilizada. La anestesia local utilizada fue la Xilocaína al $0.5 \%$ e. agua destilada, siendo aplicada por el mismo cirujano. Las demás fueron aplicadas por el anestesista. Los porcentajes fueron como lo indica el cuadro siguiente:

\section{TIPO DE ANESTESIA Y NUMERO DE CASOS}

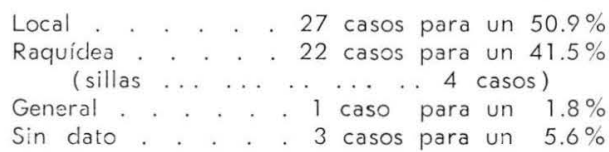

En las pacientes en las que pude participar en forma activa, las molestias durante el acto operatorio con anestesia local, se debieron más que todo a la posición adoptada para la intervención. En muy raros casos fue necesaria la colaboración del anestesista para suministrar alguna analgesia.

Técnica quirúrgica. Después de un lavado minucioso de la mucosa vaginal y toda la zona vecina, se proceclió a practicar el tacto bajo anestesia de rutina, con el objeto de descartar cualquier anomalía que hubiera pasado por alto en el examen ginecológico corriente.

Con el cuello pinzado y traccionado se calcula el rectángulo de mucosa que se va a resecar y que generalmente comprende unos 607 centímetros de largo por 4 o 5 de ancho.
Con un bisturí se marca el área rectangular comenzando a un centímetro por encima del orificio externo del cérvix y extendiéndose hasta 2 centímetros del reborde muco-cutáneo de la horquilla. En la misma forma se marca por la cara anterior, llegando hasta un centímetro del meato uretral. Se procede entonces a resecar la mucosa de izquierda a derecha, con bisturí o tijera, apoyando sobre los dedos para evitar lesiones del recto o vejiga. La hemostasis debe ser rigurosa.
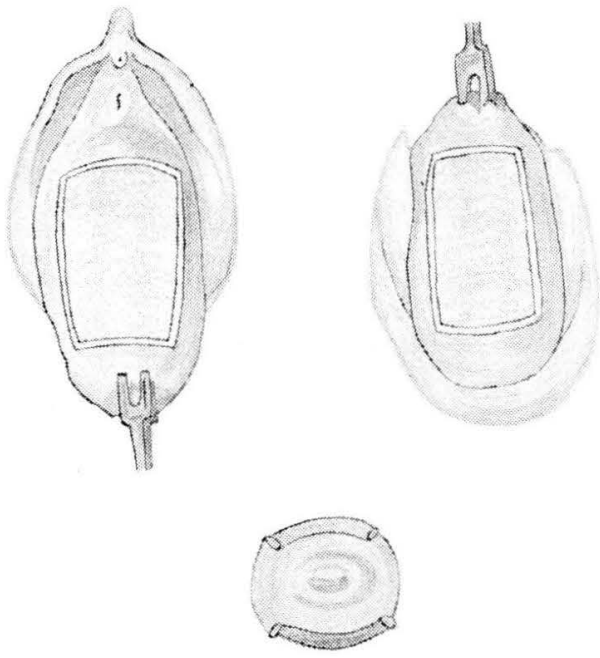

FIGURA 1 - Rectángulo sobre la pared vagina! anterior.

FIGURA 2 - Rectángulo sobre la pared vaginal posĩerior.

FIGURA 3 - Cuello uterino con su superficie de resección.

Es muy importante practicar primero la resección del rectángulo de la pared posterior con el objeto de evitar que la sangre gotee al campo operatorio, en el caso de que se comience por la pared anterior. En estas condiciones nos quedan dos bandas laterales y una transversal de mucosa indemne, que va a servir para confeccionar los túneles laterales y cervical inferior al suturar entre sí 


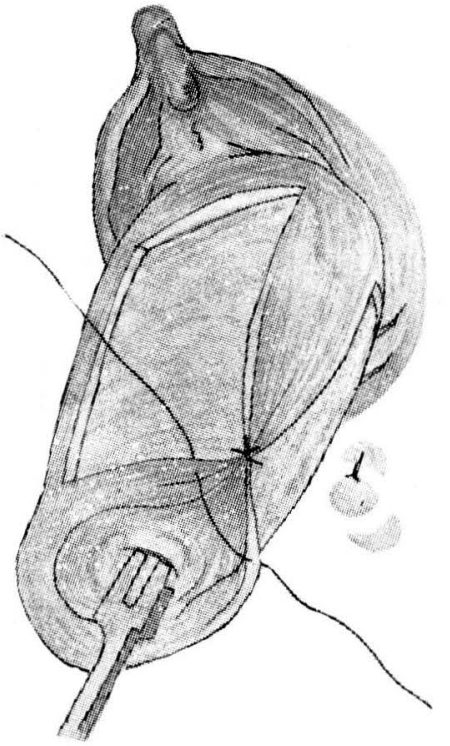

FIGURA 4 - Iniciación de la sutura en el tabicamiento vaginal.

los bordes de dicha mucosa restante. Si se cree necesario se pueden pasar unos puntos hemostáticos y de mejor afrontamiento de las superficies cruentas. Se procede entonces a la reducción de la masa prolapsada quedando entonces confrontados los bordes de la mucosa yuxta-uretral y el próximo al borde muco-cutáneo de la horquilla, los cuales se suturan dejando la vagina sellada. Para complemento de la corrección se practicará la perineorrafia sin olvidar la miorrafia de los músculos elevadores del ano (7).

Accidentes operatorios. No fue registrado alguno.

Pérdida sanguínea. Osciló entre 50 y 100 cc., sin requerimientos durante el acto operatorio.

Tiempo quirúrgico. Aproximadamente 40 minutos.

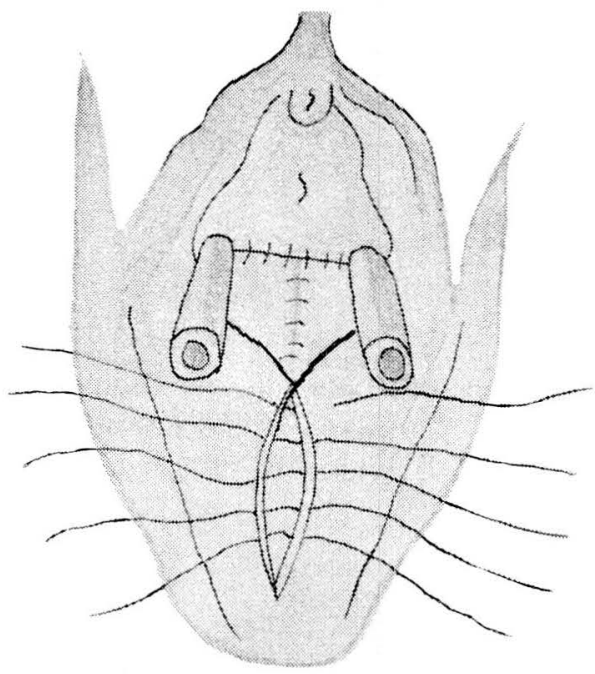

FIGURA 5 - Tabiques vaginales y colpomioplastia.

Post-operatorio inmediato. Dentro de límites normales, excepto en una paciente que murió a las pocas horas. (Este caso será referido como especial).

Cirugía sobreagregada. A una paciente de 70 años se le practicó resección de recto por procidencia grado IV. Paciente de 66 años a la que se le practicó resección de recto sigmoide con anastomosis término terminal de sigmoide del ano, por procidencia rectal gardo IV. Estadía post-operatoria de estos 2 casos, 13 y 14 días. Anestesia raquídea y general respectivamente).

En dos pacientes se practicó raspado viterino y en una de estas polipectomía. (Anatomía patológica negativa para C. A.). 


\section{COMPLICACIONES EN POST-OPERATORIO} TARDIO

Neumopatía ... Infección perineorrafia ... ........ 1 caso Hemorragia en el primer día ... ... l caso Retención urinaria pasajera ... .... 1 caso TOTAL: 4 casos para un $7.5 \%$

Esta morbilidad puede considerarso como mínima si tenemos en cuenta la utilidad de la intervención. Después de todo, cualquier tipo de intervención quirúrgica por pequeña que sea tiene algún índice de complicación.

\section{MEDICACION POST-OPERATORIA}

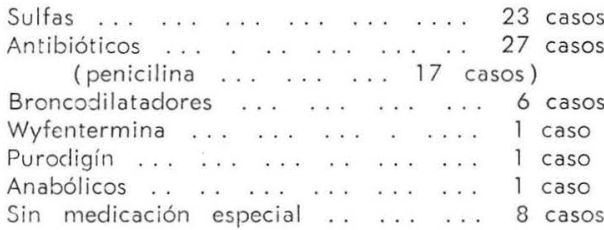

Los líquidos fueron administrados de acuerdo con las necesidades de cada paciente.

En la mayoría de las pacientes se administraron antibióticos o sulfamidas, pero no directamente por la intervención quirúrgica, sino porque como ya hemos mencionado son pacientes que padecen otras enfermedades asociadas, que sí requieren de cierta protección en el post-operatorio.

Permanencia posî-operatoria. La gráfica siguiente es muy demostrativa de la corta estada post-operatoria, si tenemos en cuenta que las pacientes más demoradas fueron las de cirugía sobreagregada. (13 y 14 días).

El promedio de permanencia sobre 49 casos es del 6.9 días. Tres de las historias no ofrecen este dato. La paciente fallecida duró aproximadamente 10 horas.

Este promedio indica la escasa morbilidad de la operación y la rapidez con que pueden abandonar el medio hospitalario, pero también deja entrever la prisa con que se autoriza la salida, sin tener en cuenta la inferioridad de condiciones a que puede someterse la paciente en su domicilio.

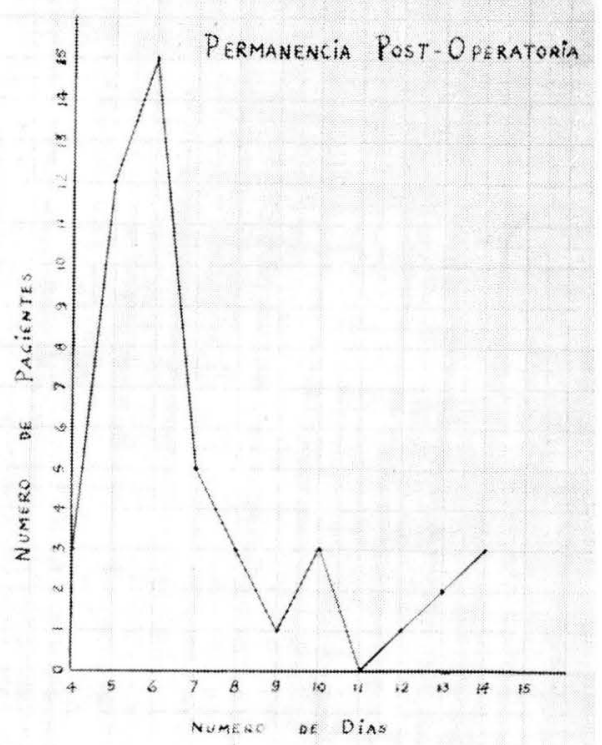

Causa de salida. Todas se dieron de alta por mejoría, a excepción de la paciente que murió. Algunas historias aparecían con causa de salida por curación, pero yo considero que no se puede hablar de curación en el post-operatorio, por lo que me he permitido utilizar la palabra mejoría on todos los casos.

Controles. Se hace sobre 51 historias, pues una paciente es de operación reciente y una figura como muerta.

\section{CONTROLES SATISFACTORIOS}

De 1 a 5 meses ... ... ...... 21 casos

De 6 a 12 meses ... ......... 5 casos Sin tiempo conocido .......... 3 casos TOTAL: 29 casos para un $56.8 \%$ 


\section{RECIDIVAS}

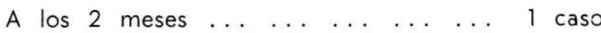

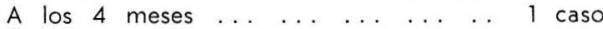

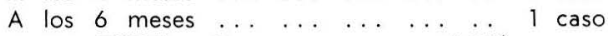
TOTAL: 3 casos para un $5.8 \%$

\section{SIN CONTROL}

Número de casos: 19 para un $37.2 \%$

Las recidivas y su tratamiento en orden, fueron las siguientes:

I. Paciente de 70 años a la que se le practicó histerectomía vaginal.

II. Paciente de 61 años a la que se le practicó plastia vaginal.

III. Paciente de 60 años a la que se le practicó histerectomía vaginal.

De esto se deduce que las pacientes no fueron bien valoradas para la primera intervención o posiblemente sus condiciones generales no permitían la realización de estas últimas intervenciones, en los casos de las histerectomías.

\section{HISTORIA DE LA PACIENTE FALLECIDA.}

Mujer de 76 años, natural de Arbeláez y procedente de Bogotá, de profesión hogar.

Motivo de consulta y enfermedad actual. Hemorragia genital de carácter intermitente, desde hace aproximadamente 3 meses y sensación de cuerpo extraño vaginal de 40 años de evolución.

Entre los antecedentes generales, bocio nodular desde hace aproximadamente 30 años. Entre los gineco-obstétricos, G4 P4, atendidos por comadrona; último parto hace 32 años. Menarquia a los 15 años, ciclos 30 por 3 días. Menopausia a los 50 años. Hace 3 años presentó hemorragia genital por lo que se le practicó raspado uterino. (No figura anatomía patológica).

Examen físico. Tensión arterial 14/7, pulso 104 por minuto. Mal estado general, desnutrida y disneica. Como datos especiales: catarata en ojo derecho y tumefacción a expensas de tiroides. Estertores en campos pulmonares y ruicios cardíacos arrítmicos con refuerzo del segundo tono.

Examen ginecológico. Genitales externos de aspecto senil; al pequeño esfuerzo se aprecia procidencia total de útero y vagina, con mucosa vaginal atrófica. Al tacto, el útero y los anexos son normales, fondos de saco libres.

Laboratorio. Dentro de límites normales.

Examen radiológico. Confirma el bocio nodular, fibrosis y enfisema en campos pulmonares. La aorta se encuentra elongada y ateromatosa; cardiomegalia por crecimiento de todas las cavidades.

Concepto carcliovascular. Aunque el riesgo quirúrgico es mayor que el usual, puede intentarse la plastia vaginal, con anestesia local.

Anatomía patológica. Cervicitis crónica severa.

Mcdicación pre-operatoria. Reposo en cama $y$ curación con mecha glicerinada durante 6 días.

Diagnósticos. 1. Prolapso genital grado III. 2. Cardioangiossclerosis. 3. Fibroenfisema pulmonar. 4. Bocio multinodular.

Premedicada la paciente se interviene, practicándole una Colpocleisis tipo Le Fort, con anestesia local de Xilocaína al $0.5 \%$, en un tiempo quirúrgico de 20 minutos, sin accidentes operatorios. Hemorragia mínima. Salida del quirófano y recuperación en buenas condiciones. A las 10 horas del post-operatorio, presenta súbito acceso disneico y muerte.

Resumen de autopsia. Muerte por edema pul. monar severo. Se plantea el interrogante de si la pacionte hizo el edema por $1.500 \mathrm{cc}$. de líquidos aplicados en 5 horas y luego la insuficiencia cardíaca aguda o si fue ésta lo primero que se presentó.

Anatomía patológica de la pieza quirúrgica. Fragmentos de vagina.

\section{VENTAJAS Y DESVENTAJAS}

Ante todo quiero anotar que la técnica de colpocleisis tipo Le Fort solo tendrá aplicación en pacientes de 
edad avanzada, con diagnóstico de prolapso genital grado III y en quienes la intervención usual puede ser fatal o dar una morbilidad marcada a causa de las condiciones médicas asociadas. Sobra decir que serán infértiles y sin vida sexual activa (8).

\section{VENTAJAS}

1. Se puede practicar con anestesia local, regional o general superficial.

2. Ha sido aceptada la técnica quirúrgica por la mayoría de los ginecólogos.

3. Es un procedimiento rápido y fácil de realizar.

4. La pérdida sanguínea es muy escasa y no se ha mencionado shock.

5. La creación de los canales transversal y laterales, permiten un buen drenaje de las secreciones.

6. Permite la deambulación el primer día post-operatorio.

7. No es necesario otro tratamiento fuera del aseo local si su patología asociada no lo requiere.

8. La práctica de la intervención se hace posible pese a la múltiple patología asociada en la paciente senil.

\section{DESVENTAJAS}

1. Inaccesibilidad del útero en casos de hemorragia post-menopáusica.

2. Dificultad en el diagnóstico de carcinoma uterino después de la intervención.

3. Imposibilidad para realizar el acto sexual.

Dados estos factores, el cirujano deberá asegurarse de que la paciente no esté recibiendo hormonas capa- ces de provocar una hemorragia benigna post-menopáusica.

Una citología de rutina previa a la intervención así como un raspado fraccionado en caso de sospecha de C. A. son absolutamente indispensables.

Con relación al coito es de suponer que todas estas pacientes ya no tienen vida sexual activa y que será cle discutir si es más importante para ellas, evadirse de esa gran incomodidad de portar un cuerpo extraño que procurar una vagina funcionante que muy seguramente no va a utilizar.

De todas maneras, hago hincapié en que nuestra obligación es la de comunicar la limitación funcional antes de intervenir y esperar la autorización por parte de los cónyuges; pues de lo contrario nos podríamos encontrar en situaciones muy desagradables.

\section{RESUMEN Y CONCLUSIONES}

1. Se analizan detalladamente 53 casos de colpocleisis de Le Fort practicados durante los últimos diez años en el servicio de Ginecología y Obstetricia del Hospital San Juan de Dios de Bogotá. En la introducción al trabajo se hace una reseña histórica y de técnica quirúrgica.

2. La selección de las pacientes se basó en la edad y las condiciones médicas asociadas, que hacían necesario un procedimiento quirúrgico poco traumático. El promedio de edad fue de 72.83. En $69.8 \%$ de los casos estaba presente la arterioesclerosis sola o asociada (39.6\%) con cardiopatía; el riesgo quirúrgico por el aspecto cardiovascular estaba aumentado en el $54.3 \%$ del grupo en estudio. En el $81 \%$ de los casos se haIlaron afecciones broncopulmonares 
crónicas. En contraste solo 9 casos $(16.9 \%)$ presentaron patología ginecológica benigna concomitante al prolapso.

3. Se describe la técnica quirúrgica utilizada, mostrando los detalles importantes para su correcía realización, se insiste en la necesidad de la colpoperineorrafia posterior con miorrafia de los elevadores.

4. La anestesia utilizada fue la local en el $50.9 \%$ y la regional en el $41.5 \%$, solo en un caso se administró general. El tiempo quirúrgico promedio se estimó en 40 minutos y la pérdida sanguínea entre 50 y $100 \mathrm{cc}$. El promedio de permanencia hospitalaria post-operatoria fue de 6.9 días para 49 pacientes. En dos casos de cirugía agregada por procidencia rectal fue de 13.5 días. Solo 4 casos $(7.5 \%)$ presentaron morbilidad postoperatoria. Un caso ( $1.8 \%$ ) murió en el post-operatorio inmediato por causa indirecta a la intervención misma. En los resultados a largo plazo se hallaron 3 recidivas del prolapso, sobre 32 casos que regresaron a control.

5. Se enumeran las ventajas e inconvenientes de la intervención, insistiendo sobre la necesidad de hacer saber a la paciente la imposibilidad para efectuar el acto sexual.

\section{BIBLIOGRAFIA}

$1 \mathrm{AMOROCHO}, \mathrm{C} . \mathrm{J} .$, Comentarios sobre los prolapsos genitales. Col. Med. Bogotá 4 (12) 446-451 Septiembre 45 .

2 APARICIO, J. A. Comentarios sobre 154 casos de prolapso genital. Rev. Col. Obs. y Ginecol. Bogotá, 4 (4) 129-139. Jun. 53.

3 CARDONA, C. P. N. y otros. Tratamiento quirúrgico del prolapso genital. Ant. Med. Medellín. 3: 374-407. Jun. 53.

4 DAVIS, C. H. and CARTER, B.: Gynecology and Obstetrics. Edit. W. F. Prior. CO. Hagerstown. 1958. 3, 76-84.

5 EMGE, C. A. Correction of Genital Prolapse of the Elderly. Am. J. Obst. Gynec. 95: 362, Junio 1966.

6 GREENHILL, J. P.: Cirugía ginecológica. Edit. Interamericana, S. A. México 1959. Primera edición. pp. 184-187.

7 HEINRICH MARTIUS. Operaciones ginecológicas. Edit. Labor, S. A. Barcelona-Madrid. 1957. Tercera edición. pp. 269-271.

8 HENRY C. FALK, and SHERWIN A. KAUFMAN, M. D. Partial Colpocleisis: The Le Fort procedure. Analysis of 100 cases. Obstetrics and Gynecology 5, Number 5, 617-28 Mayo 1955.

9 MENDEZ, L. M. y PUTMAN T. E. La operación de Le Fort en prolapso uterino senil. Rev. Med. Col. Barranquilla 3 (12) extraord. 3: 374-407. Jun. 53.

10 NOVAK-SEEGAR, Tratado de Ginecología. Edit. Interamericana S. A. México 1962. Sexta edición. p. 287.

11 SANCHEZ, L. A. Tratamiento quirúrgico de los prolapsos uterinos. Edit. HAZ. Madrid, 1941. pp. 273-281.

12 Suarez, R. B. Prolapso genital. Col. Med. Bogotá 3 (3) 70-74. Dic. 43. 